\title{
Estado de moralidad/inmoralidad de una escuela de medicina y un hospital público: aplicación de una estrategia de moralización
}

\author{
Morality/immorality status of a school of medicine and a public hospital: application of \\ a moralization strategy
}

\author{
Alberto Perales ${ }^{1}$, Pedro Ortiz ${ }^{1}$, Violeta Nolberto ${ }^{2}$ \\ ${ }^{1}$ Instituto de Ética en Salud. Facultad de Medicina, Universidad Nacional Mayor de San Marcos. Lima, Perú. \\ 2 Facultad de Matemáticas, Universidad Nacional Mayor de San Marcos. Lima, Perú.
}

\section{Resumen}

Objetivos: $1^{\circ}$ ) Evaluar en los profesores de la Escuela de Medicina de la UNMSM el nivel de conocimientos sobre ética, deontología y bioética clínica; $2^{\circ}$ ) Identificar los problemas éticos más frecuentes en la relación docente-discente y aquellos en las sedes docentes; $3^{\circ}$ ) Con los resultados, construir un seminario-taller el cual, una vez validado, se ofreciera al profesorado como enseñanza básica; $4^{\circ}$ ) Contribuir a la moralización institucional, reforzando el ethos institucional. Lugar: Escuela de Medicina, Facultad de Medicina, Universidad Nacional Mayor de San Marcos (UNMSM), Lima, Perú. Métodos: Para los objetivos $1^{\circ}$ y $2^{\circ}$, estudio transversal, descriptivo, con metodología cualitativa y cuantitativa. Se seleccionó 26 profesores y 2 autoridades, de acuerdo a cinco criterios, para realizar entrevistas en profundidad. Sobre los resultados cualitativos se construyó y validó un cuestionario específico. Se aplicó el cuestionario a una muestra seleccionada por muestreo probabilístico sistemático, que representaba al $29 \%$ del total de 801 profesores de la Escuela de Medicina. Para el objetivo $3^{\circ}$, se utilizó un estudio comparativo pre y post. Para el $4^{\circ}$ objetivo, ya validado el curso, se aplicó el seminario-taller y se midió el grado de satisfacción de los participantes. Resultados: En cuanto a los objetivos $1^{\circ}$ y $2^{\circ}$, se identificó diversos problemas éticos en la relación docente-alumno, entre los mismos profesores y en la práctica profesional hospitalaria, que vienen generando modelos inadecuados de atención profesional y afectando la formación moral del estudiante. Entre los resultados cuantitativos se precisó que, del total de profesores, $59 \%$ no había llevado curso formal alguno sobre ética, deontología o bioética; solo $6,3 \%$ había llevado cursos específicos sobre este tema, a nivel de posgrado; $84 \%$ no conocía adecuadamente cuáles son los principios de la Bioética; $53 \%$ no conocía adecuadamente cuáles son las funciones de un Comité Hospitalario de Ética; $58 \%$ no conocía adecuadamente cuáles son las funciones de un Comité de Ética de Investigación; y $71 \%$ no conocía adecuadamente el Código de Ética del Colegio Médico del Perú. En relación al objetivo $3^{\circ}$, el seminario-taller fue validado y probado en su eficacia en dos sedes docentes $(p=0,001$ en la prueba de Levene; $y, p=0,000$ en la prueba de Mann Whitney), respectivamente. Y en el objetivo $4^{\circ}$, el seminario fue impartido a 296 profesores, habiéndose convertido, en la actualidad, en un Seminario permanente de adiestramiento básico en ética y deontología en salud y bioética clínica. Finalmente, el curso fue solicitado por una institución hospitalaria de salud para ser aplicado a todo su personal, con el propósito de disminuir los serios conflictos éticos que existían y amenazaban su ethos institucional. Conclusiones: 1) El deficiente nivel de conocimiento sobre ética, deontología y bioética clínica de los profesores de la Escuela de Medicina de la UNMSM fue comprobado y mejorado. 2) Se identificó los problemas morales institucionales fundamentales. 3) El Seminario-Taller construido fue validado y demostró su eficacia en dos sedes hospitalarias, con diferencias estadísticamente significativas en diseño pre y post. 4) El $97 \%$ de los profesores que recibió el curso declaró que los había ayudado entre mucho y muchísimo. 5) El método utilizado aplicado (por expresa solicitud) a un hospital público abre interesantes perspectivas como estrategia de moralización institucional y disminución del estrés laboral.

Palabras clave: Ética profesional; bioética; ética clínica; deontología; ética institucional.
Abstract

Objective: $1^{\circ}$ ) To evaluate San Marcos University School of Medicine Faculty professors' knowledge on ethics, deontology and clinical bioethics; $2^{\circ}$ ) To identify the most frequent ethical problems in both professor-student relationship and teaching hospitals; $3^{\circ}$ ) With the results obtained to construct a workshop which, after being validated, could be offered to the whole faculty as a basic course; $4^{\circ}$ ) To contribute to the institutional moralization by reinforcing the institutional ethos. Setting: School of Medicine, Faculty of Medicine, Universidad Nacional Mayor de San Marcos (UNMSM), Lima, Peru. Methods: For objectives first and second, transversal and descriptive design, with qualitative and quantitative methodologies. Following a pre selected criteria 26 professors and 2 Faculty authorities were selected to be interviewed in depth. Upon the qualitative results a specific questionnaire was constructed and validated. Thereafter it was applied to a professor's sample selected by means of systematic probability sampling that represented $29 \%$ of the whole faculty, 801 professors. For the third objective, a pre-post comparative study was used. For the fourth objective, a validated workshop was applied and afterwards the participants' satisfaction degree was evaluated. Results: Regarding to objectives first and second, diverse ethical problems were identified in the professorstudent and among the professors relationships; also related to hospital professional practice which were generating inadequate health care models and affecting the medical students' moral training. Among the quantitative results $59 \%$ of professors had never received a formal course on ethics, deontology or bioethics; only $6,3 \%$ had received them at post-graduate level; $84 \%$ did not adequately know which were the Bioethical principles; $53 \%$ did not adequately know the Hospital Ethical Committee's functions; $58 \%$ did not adequately know the Research Ethical Committee' functions, and $71 \%$ did not adequately know the Peruvian College of Physician's Code of Ethics. Regarding the third objective, the workshop was validated in 2 teaching hospitals (Levene: $p=0,001$, and Mann Whitney: $p=0,000$ ) respectively. Regarding objective fourth, the workshop was given to 296 professors. Currently it has been instrumented as a permanent basic training course on ethics and deontology in health and clinical bioethics. Finally, the course was requested by a public hospital to be applied to its whole personnel as an attempt to disminish the serious ethical conflicts that were affecting the institutional ethos. Conclusions: 1) San Marcos University School of Medicine faculty's deficient knowledge on ethics, deontology and clinical bioethics was verified and subsequently improved. 2) The most important institutional ethical problems were identified. 3) The wokshop constructed upon the results was validated in two teaching hospitals and showed significant statiscal differences in pre and post design. 4) $97 \%$ of the professors receiving the course declared to have been helped much and very much. 5) The method was also applied (upon special request) to a public hospital, opening interesting perspectives as a new strategy for institutional moralization and to diminish work stress.

Key words: Ethics, professional; bioethics; ethics, clinical; deontology; ethics, institutional. 


\section{INTRODUCCIÓN}

Los cambios en la nueva estructura económica globalizada de la sociedad y la presión de retorno de la inversión realizada en desarrollo tecnológico, viene reflejándose en nuestra sociedad como una tendencia a comportamientos y actitudes deshumanizadas en todos los campos de la actividad del hombre ${ }^{(1)}$. El área de la atención médica y de salud, como terreno específico en el cual se trata el dolor humano, constituye escenario preferencial para lamentar tales características. Inevitablemente, tal influencia afecta también a las instituciones formadoras de recursos humanos en este campo. La deshumanización del 'acto médico' se evidencia con intensidad creciente en las instituciones del Ministerio de Salud y de la Seguridad Social, forzadas a una labor asistencial con altos índices de concentración y escasos recursos ${ }^{(2)}$. El problema se agrava cuando empresas privadas convierten la medicina en una inversión comercial que busca rentabilidad (3), en el cual imperan las leyes del mercado (4). En tal sentido, una formación médica científico-humanista y un énfasis temprano en una cultura de valores deben ser, a la par que una sólida base para la formación profesional, los ejes centrales del desarrollo de actitudes morales en los nuevos profesionales médicos, que trabajarán en un medio social cada vez más contradictorio, en razón de las presiones tecnológicas y económicoutilitaristas en ascenso continuo.

Lo anterior supone el imperativo moral de las instituciones formadoras de asegurar que los educadores posean los conocimientos necesarios de ética, deontología y bioética, no solo para formar a los nuevos profesionales en perspectiva integral, sino para contribuir a optimizar el ethos institucional que como ambiente colectivo contribuye diariamente a crear la atmósfera de paz y respeto necesaria para la adquisición de actitudes profesionales adecuadas. Los alumnos no solo aprenden de la información científica y tecnológica que se les imparte ${ }^{(1)}$ sino, mucho más aún, de lo que observan en el proceder técnico y la conducta profesional de sus profesores y autoridades.

El riesgo de un contraste negativo entre lo aprendido teóricamente en las aulas en materia de ética y lo observado en las prácticas realizadas en instituciones de salud, es decir, en la cruda realidad de los hospitales, puede convertir tal experiencia de aprendizaje en estímulo deformador en vez de formador, como se desea. Postulamos, así, que un sistema generador de futuros médi- cos, sin sólidas bases éticas, corre alto riesgo de deshumanizarse y contribuir -sin proponérselo intencionalmente-a consolidar una medicina contraria a las necesidades reales de la sociedad a la que pertenece.

Por otro lado, desde hace más de una década se viene dictando cursos de Bioética en la Facultad de Medicina de la Universidad Nacional Mayor de San Marcos (UNMSM), con el propósito de capacitar al estudiante en tal materia. Los profesores de todas las áreas de la Escuela de Medicina, sin embargo, no necesariamente han seguido cursos de capacitación específica en la materia, de modo tal que el sistema en conjunto, docente-discente, puede no estar hablando el mismo lenguaje ético imprescindible para la construcción del 'ethos institucional'.

Sobre la base de considerar a la institución formadora, en este caso, la Escuela de Medicina de San Fernando, como un sistema ${ }^{(5)}$ educativo, el presente estudio, intenta producir no solo conocimiento que contribuya a la comprensión de esta problemática, sino a crear una estrategia práctica de moralización del sistema global seleccionado y contribuir a tal finalidad. Para ello, se elaboró un proyecto de 5 años y medio de duración. El primer año y medio, fue llevado a cabo de agosto 2002 a noviembre 2003, sin apoyo económico de la universidad; y los restantes, de 2004 al 2007, con financiamiento de la misma.

Los objetivos del presente estudio tuvieron varios niveles de complejidad:

$1^{\text {o }}$ Evaluar el nivel de conocimientos y la formación específica que sobre ética, deontología y bioética clínica tienen los profesores de la Escuela de Medicina de la UNMSM.

2o Identificar los problemas éticos más comunes en la relación docente-discente en las sedes docentes de la Facultad

3ํ Sobre la base de tal información, elaborar un Seminario-Taller en ética, deontología y bioética clínica que, una vez validado, se impartiera a los profesores como esquema teórico-práctico para la enseñanza básica en esta materia, que cubriera las necesidades detectadas.

$4^{\text {o }}$ Contribuir a la moralización de la institución, reforzando el 'ethos institucional', con aplicación de una estrategia de moralización.

\section{MÉTODOS}

Estudio transversal descriptivo, en una población de 801 docentes, cuyos primeros dos objetivos se cumplieron con metodologías de investigación cualitativa y cuantitativa. Para el enfoque cualitativo, del universo de profesores se seleccionó 26 'informantes clave', utilizando los siguientes criterios de heterogeneidad: a) Departamento académico en que ejercía la docencia; b) Edad: el mayor y el menor de dicho departamento; c) Sexo: hombre y mujer; d) Número de profesores por departamento, divididos en dos categorías: los grandes departamentos con más de 100 profesores y los departamentos menores con menos de 100 profesores; dos autoridades: El Secretario Académico de la Facultad de Medicina y el Director de la Escuela de Medicina.

A ellos se les practicó entrevistas en profundidad, luego de explicarles los propósitos del estudio y lograr su consentimiento informado para participar. El análisis de contenido de las entrevistas permitió la identificación de áreas de conflicto ético, en base a las cuales se diseñó un instrumento de encuesta, para ser aplicado a la población objetivo, con metodología cuantitativa (preguntas cerradas) y cualitativa (preguntas abiertas).

Dicho cuestionario cubrió las siguientes áreas: principios, relación médico-paciente, consentimiento informado, problemas éticos del comienzo de la vida, problemas éticos del final de la vida, Comités de Ética Hospitalaria, Comités de Ética de Investigación, Código de Ética del Colegio Médico, enseñanza de la ética, problemas éticos de la relación docente-discente y tres preguntas abiertas: (a) Liste los tres problemas éticos más frecuentes que encuentra en su especialidad, (b) Liste las causas más importantes que, en su opinión, generan los problemas que Ud. señala, (c) Qué sugerencias aportaría Ud. para mejorar la enseñanza de la ética en nuestra Facultad.

El cuestionario fue aplicado, mediante muestreo no probabilistico voluntario (previo consentimiento informado), a 232 docentes (29\% del total). Los datos fueron tabulados por distribuciones de frecuencias simples bivariadas. Siendo el objetivo especificamente descriptivo, no se aplicó pruebas estadísticas diferenciales.

\section{RESULTADOS}

\section{Cuantitativos:}

De los 801 profesores de la Facultad de Medicina de la UNMSM, en relación al sexo $80 \%$ fue masculino y $20 \%$, femenino. La edad promedio fue 54,8 años; el promedio de años de experiencia docente fue mayor a 10 años; 85\% tenía profesión médica y $15 \%$ pertenecían a otras profesiones. 
Luego que la información propocionada por la encuesta fuera sometida a análisis estadístico descriptivo, se obtuvo los siguientes resultados: 59\% no llevó curso formal alguno sobre ética, deontología o bioética; solo 6,3\% había llevado cursos específicos sobre este tema, a nivel de posgrado; $84 \%$ no conocía adecuadamente cuáles eran los principios de la Bioética; $53 \%$ no conocía adecuadamente cuáles eran las funciones de un Comité Hospitalario de Ética; $58 \%$ no conocía adecuadamente cuáles eran las funciones de un Comité de Ética de Investigación y $71 \%$ no conocía adecuadamente el Código de Ética del Colegio Médico del Perú.

\section{Cualitativos:}

Estos fueron agrupados por niveles de convergencia.

1. Problemas más generales: negocio de trasplantes; aborto; ligadura de trompas; deshumanización de la medicina; complicidad "las faltas se pasan por alto"; valorar la carrera por la fama y el dinero.

2. Relaciones interpersonales: celos entre profesionales. Pésimas relaciones entre médicos y obstetrices y otros profesionales; se habla mal del colega delante del paciente; hay maltrato al Interno; malas relaciones entre docentes; hay acoso sexual a las Internas e impuntualidad en todas las actividades.

3. En la administración: se usa la jefatura autoritariamente, maltratando al personal subalterno; los médicos administrativos maltratan a los asistenciales.

4. En la docencia - profesores: impuntualidad; expresarse mal del colega ante el alumnado; no hay enseñanza de actitudes correctas; participación en varias universidades; no hay verdadera vocación docente; conducta motivada por interés crematístico.

5. En la docencia - alumnos: indisciplina e impuntualidad; piden postergación de exámenes sin razón; conducta deficitaria en valores; motivación crematística en la carrera; han habido algunos casos de conducta delictiva.

El tercer objetivo se cumplió construyendo un Seminario-Taller, sobre la base de los resultados y utilizando el encuadre teórico de Pedro Ortiz (1997) sobre el desarrollo moral del hombre.

El Seminario -Taller fue diseñado incialmente para un dictado que cubría 3 sesiones de 6 horas cada una, que se cumplía en tres días consecutivos. La irregularidad de la asistencia continua de los profesores, sin embargo, obligó a comprimir el curso en ediciones sucesivas, hasta que fue dictado en una sola sesión de 6 horas, que se dicta en una mañana. El contenido del Seminario Taller fue sometido a validación, con pruebas de entrada y salida, aplicadas al personal docente de dos sedes hospitalarias de la Facultad (sede 1 y sede 2). Para tal validación, la muestra fue no probabilística voluntaria. La prueba de entrada fue anónima, establecida por grupo cerrado. De ellos, por motivo de carga asistencial, algunos declinaron de participar en la prueba de salida, que se realizaba en la segunda parte del Seminario, dictado en día diferente. En la sede 1, participaron 34 en la prueba de entrada y 27 en la de salida. Se obtuvo 10 y 14,59 de puntaje, lográndose un incremento de 4 puntos, que resulta un claro indicador de efectividad ( $\mathrm{p}=0,001$ en la prueba de Levene). En la sede 2, se procedió con la misma metodología, obteniéndose 11,04 en la prueba de entrada y 15,06 en la de salida ( $p=0,000$ en la prueba de Mann Whitney). La comparación entre ambos hospitales deja una diferencia de 1 punto a favor de la sede 2, en donde se observó una participación mucho más activa de las autoridades del hospital. De este modo, el estudio comprueba la hipótesis planteada (Figuras 1 y 2).

El cuarto objetivo se cumplió con la extensión de la aplicación del curso.

En base a estos resultados, el Seminario-Taller se siguió aplicando en el 2005 y 2006, a los profesores de otras sedes y departamentos académicos de la Facultad. El nivel de satisfacción se evaluó en una escala de Likert de 5 puntos ( $1=$ Totalmente insatisfecho, (No me ha ayudado); $2=$ Insatisfecho, (Me ha ayudado poco); $3=$ Moderada insatisfacción, (Me ha ayudado medianamente), $4=$ Satisfacción, (Me ha ayudado mucho); $5=$ Completa satisfacción, (Me ha ayudado muchísimo). El $36 \%$ de los profesores opinó que el Seminario-Taller los había ayudado 'muchísimo' (máximo puntaje de la escala) y $64 \%$, 'mucho', que sumados alcanzan el 97\% de satisfacción. Además, recomendaron ampliar el número de horas, darle creditaje y dictarlo en modalidad obligatoria, en coordinación con las autoridades de la Facultad de Medicina.

Tal recomendación se llevó a cabo en el 2007, como actividad

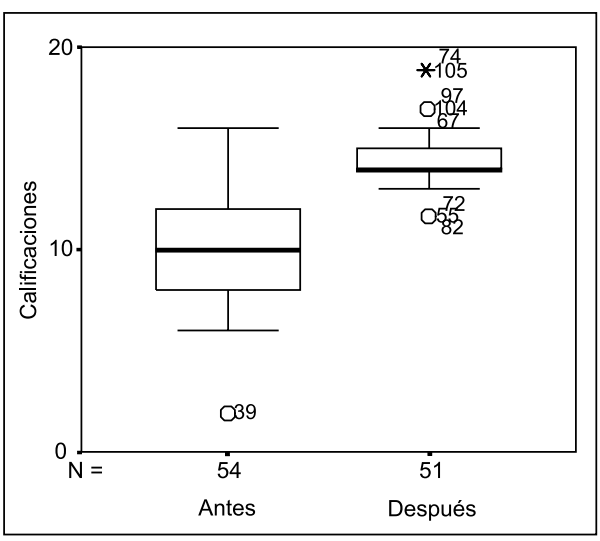

Figura 1. Gráfico de cajas de los resultados de la sede 1. Después de la intervención, los participantes incrementaron su rendimiento: la mediana es mayor (14) respecto a 10; así mismo, el rendimiento es más homogéneo (prueba de Levene).

válida para el proceso de ratificación y promoción docente de la institución. Aún así, la carga docente y, particularmente una larga huelga de profesores y administrativos, por reclamos salariales, impidió que pudiera impartirse a todos los profesores. Por tal razón, el curso ha pasado a ser actividad permanente del Instituto de Ética en Salud. Más aún, a pedido del personal administrativo, se ha ampliado el dictado del Seminario a este sector. En resumen, el total de profesores adiestrados fue 296.

\section{Impacto externo del proyecto: aplica- ción a un hospital público}

Preocupados por la presencia de problemas éticos y laborales que frustraban los esfuerzos de desarrollo institucional y creaban un clima laboral tenso y no saludable, tanto para el personal en general como para los pacientes en particular, el Cuerpo de Gestión de un Hospital Público nos solicitó el curso para mejorar su ethos institucional. Los objetivos específicos fueron:

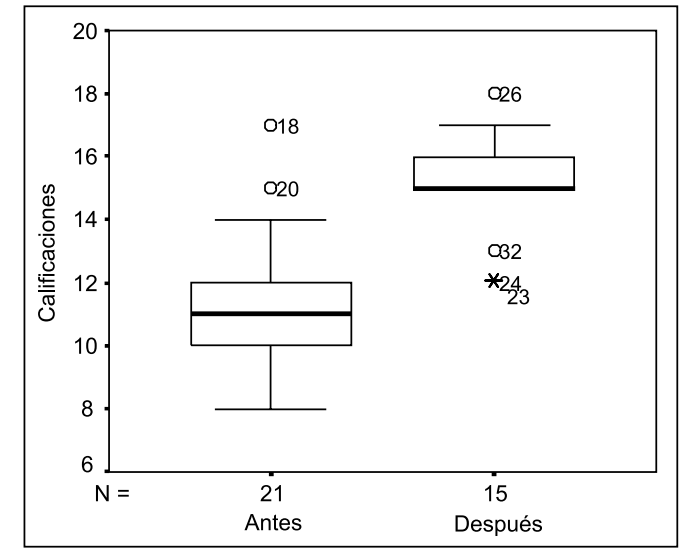

Figura 2. Gráfico de cajas de los resultados de la sede 2. Después de la intervención, los participantes incrementaron su rendimiento; la mediana es mayor (15) respecto a 11; así mismo, el rendimiento es más homogéneo (prueba de Mann Whitney). 
a) Desarrollar competencias para identificar, analizar y dar adecuada solución a los problemas éticos que se suscitaban en la práctica clínica y administrativa.

b) Reflexionar sobre las actitudes personales ante los problemas descritos.

c) Motivar el desarrollo de una cultura ética 'en el Hospital'.

El Seminario-Taller fue dirigido a todo el personal de la institución, tanto profesional como administrativo. Desde sus autoridades máximas hasta los niveles de menor rango participaron en calidad de alumnos.

El método utilizado fue interactivo, por grupos de 30 miembros, en promedio, utilizándose exposiciones teóricas, dinámica de grupo y talleres de análisis de problemas éticos en situaciones clínicas y administrativas. Cada grupo recibió el curso en una sesión de 6 horas. La actividad se realizó en los ambientes del pabellón de consulta externa del propio hospital, del 18 de octubre, 2005, al 16 de febrero de 2006.

En la organización y ejecución de la actividad se contó con el apoyo de la Dirección y de la Oficina de Personal, así como con el soporte de las Unidades Técnica computarizada y de Imagen de la institución.

Como resultados, asistieron 865 participantes: 692 miembros del personal nombrado y 177 contratados.

En la prueba de entrada, el promedio de porcentaje de respuestas correctas fue $25 \%$ y en el de salida, 57\%. Siendo idénticas, permite señalar que se duplicó el nivel de conocimientos de ética, deontología y bioética clínica del personal al final del curso.

Al mismo tiempo, con las preguntas abiertas del cuestionario se captó información sobre diversos problemas que en opinión del personal incidían en los conflictos organizacionales que enfrentaban. A continuación se los lista:

1. Identificación del personal con la institución: No conoce la historia de la institución; no conoce los objetivos institucionales; en su mayoría, prioriza sus objetivos personales sobre los institucionales; el trabajador "solo responde por su turno"; exige derechos, pero no deberes; cualquier beneficio o cambio temporal lo transforma en un derecho permanente; busca dar lo menos posible a la institución ("¿A qué hora salgo y me voy a hacer lo mío?”).

2. Corrupción: Hay robos en todos los Servicios; se lucra con consultas particulares y hay quien cobra a los pacientes; se sustrae alimentos; hay descansos médicos no justificados; hay coimas.

3. Violación de los derechos de los pacientes: No se les informa adecuada ni oportunamente sobre su condición; no se les informa sobre la normatividad institucional; no se les pide consentimiento informado; no se guarda el secreto profesional. Se comenta abiertamente sus problemas personales; se les pone sobrenombres; cuando un paciente tiene un accidente, se oculta información; hay maltrato al paciente y a sus familiares; se cita al paciente a una hora, mientras que el/la profesional llega tarde y lo atiende tardíamente. Pero si el/la paciente llega tarde, se le castiga dejándolo sin atención

4. Incompetencia y necesidad de capacitación: Hay jefaturas que son asignadas por amistad y no por concurso de méritos; hay jefes que no tienen el profesionalismo necesario para el cargo; hay jefes que carecen de conocimientos adecuados; hay jefes que violan los derechos de los pacientes y del personal subalterno; algunos jefes salen a tomar licor con miembros de su personal: el personal profesional y administrativo necesita capacitarse y actualizarse.

5. Malas relaciones interpersonales: Hay maledicencia; hay agresiones entre miembros del personal; hay relaciones sentimentales adúlteras; el chisme es maligno y afecta las relaciones laborales; no hay solidaridad; se usa lenguaje vulgar; se usa la calumnia; hay malas relaciones entre directivos, jefaturas y personal.

6. Incumplimiento de funciones: No se pasa visita médica; se incumple el horario laboral en todos los niveles; se incumple las citas; hay impuntualidad en los cambios de turno; cuando el hospital celebra alguna festividad, se suspende la atención; no se evalúa a los pacientes con regularidad; no hay productos suficientes en la farmacia.

7. Deshumanización de la atención: Se maltrata al paciente y violan sus derechos; el paciente es visto como fuente de ingreso económico; el diagnóstico y el tratamiento no se extiende a la familia; hay manejo inadecuado de los pacientes con agitación psicomotriz; aparte de la enfermedad, no se identifica ni trabaja las otras necesidades del paciente; la aplicación de la terapia electroconvulsiva deja mucho que desear; se hace entrega del Servicio en presencia del paciente.
8. Imagen del médico: El personal espera el liderazgo médico, pero éste no da los mejores ejemplos; incumple los horarios; algunos utilizan a los pacientes como fuente de ingreso; se faltan el respeto y se ofenden entre colegas; no hay compañerismo; no hay presentaciones clínicas; la relación emocional con el paciente y el trato que se les da dejan mucho que desear; se desprestigian entre ellos frente al personal o los pacientes; faltan el respeto a sus superiores y a sus subordinados.

9. Violación de principios y valores: Se viola la Autonomía; no se ejerce la Beneficencia; hay casos de No-Maleficencia; no hay Justicia; no hay Solidaridad; no se respeta la Dignidad del paciente y, no rara vez, del personal subalterno; hay casos de falta de honradez; la impuntualidad es frecuente.

10. Miscelánea: Hay nepotismo y compadrazgo; hay un manejo psicosocial que trata de alterar el clima institucional; no hay trabajo con la comunidad; no hay programa de rehabilitación; algunos trabajadores tienen patología mental; hay pacientes con tuberculosis tratados inadecuadamente; la vestimenta del personal es inadecuada. No hay uniformes; no hay solidaridad en el cuerpo directivo.

\section{Percepción de utilidad y satisfacción con el Seminario Taller}

El 91\% de los asistentes estuvo altamente satisfecho con el seminario-taller. Solo 2\% no contestó la pregunta.

Beneficios logrados con el Seminario-Taller (comentarios de los participantes):

Para nuestra sorpresa, los participantes señalaron que los beneficios no solo fueron circunscritos sino que se extendieron a diferentes áreas y, muy particularmente, al área personal. Extraemos de una larga lista las más distintivas:

1. Beneficio personal: Me ayudó a cambiar de actitud y ser menos egoísta y más solidario; aprender sobre valores, principios éticos y bioéticos y la importancia de aplicarlos en la situación laboral y personal; tener más criterio para tomar decisiones y mejorar mi ética profesional; resolver los conflictos éticos en el trabajo; a comprender cómo afectan los problemas del trabajo mis relaciones familiares; mejorar como trabajador para prestar mejor atención a los pacientes y así mejorar a la institución; mejorar en 
el servicio en que trabajo (Emergencia), pero, a la vez, en muchos aspectos de mi vida; relajarme más porque muchas veces en el trabajo nos encontramos estresados; evidenciar el riesgo que todos tenemos de incurrir en fallas morales.

2. Beneficio de los pacientes: A cómo comunicarme con los pacientes. El vídeo ayuda a comprender la importancia de la solidaridad, el respeto y la atención que se merecen los pacientes; saber escucharlo, hacerlo sentir que es muy importante; a mejorar la entrevista médico-paciente; recordar que los pacientes también tienen derechos y necesidades; a tratar al paciente como una personalidad; a brindar confianza y levantar la autoestima del paciente.

3. Beneficio institucional: A comprender al hospital como sistema y la cultura organizacional con sus problemas propios; a comprender la importancia de la solidaridad, el respeto y la atención que se merecen los trabajadores; a identificar los valores para un cambio institucional; a valorar la confianza que se debe generar para el logro de cualquiera de nuestros objetivos; nos permitió autoevaluarnos como servidores de la institución. Cambiar actitudes; obtener esperanza de desarrollo y no visión catastrófica; al escuchar cómo piensan los demás, participar de una intervención psicoterapéutica con trabajadores de la Institución y ver nuestras semejanzas; a reflexionar sobre cómo me afectan los problemas del hospital, y que tal vez sin grandes proyectos pueda hacer algo (jefe de unidad); el análisis de la metodología frente a un problema ético y la presentación del caso clínico es donde uno aprende a cómo manejarlos; a comprender la corrupción; a definir mejor mi rol en mi institución; en tratar de mejorar para el bien de la Institución y de mí mismo; de aquí en adelante, nuestro trabajo y nuestra identificación con la Institución deben llevar al 'hospital' a ser uno de los mejores; en querer más a mi Institución; a no estar oyendo y llevando chismes; a identificar los riesgos de laborar en instituciones de Salud Mental.

4. Miscelánea: En realidad, todo el curso me ayudó mucho para poder actuar con prudencia antes de tomar una decisión; todos los trabajadores deberían recibir el curso; que teniendo voluntad y compromiso de cambio se puede hacer Patria; que los Directores sigan así, preocupándose por la Institución, con autoridad y equidad; que se desarrollen prácticas de grupo sobre acontecimientos de la actividad laboral; gracias a los directivos que organizaron el curso, por el interés para con el personal de la institución; cuando los cursos son buenos nunca es tarde para aprender más.

\section{DISCUSIÓN}

Orfila Barreto ${ }^{(6)}$ cita a Dewey, quien "formula una tesis curricular contextual, en la que destaca la pertinencia curricular a partir de los estudiantes. En esta desarrolla los supuestos filosóficos siguientes:

- El contenido curricular tiene que responder a lo que ocurre en la sociedad y estar relacionado con las experiencias de la vida.

- Las disciplinas constituyen un medio para el estudio de la sociedad y para la solución de problemas, si promueven el desarrollo del significado basado en la experiencia del estudiante.

- El aprendizaje debe ser activo, participativo y autodirigido por el estudiante.

- El currículo debe estar constituido por experiencias formuladas reflexivamente, que se relacionen con los problemas del diario vivir y que consideren situaciones pertinentes para el estudiante." Para Dewey, la experiencia consiste en la interrelación de la persona con su medio ambiente...la fundamental es la relacionada con la que comparten los grupos más amplios.......el logro de los objetivos educativos consiste en la reorganización y reconstrucción constante de la experiencia. Esta reconstrucción estriba en un proceso mediante el cual se reformula o renueva la experiencia básica del estudiante en una que éste entienda y valore...es importante la democracia participativa" (7).

Nuestro Seminario-Taller sobre Ética, Deontología y Bioética Clínica se alinea con tales postulados. Gran parte de su éxito, ahora comprendemos, depende no solo de que el contenido de su estructura se basa en los problemas reales detectados en el ambiente universitario de la Facultad de Medicina, sino en su relación con lo que ocurre en la cultura organizacional y las experiencias vitales de todos los días de los profesores de la Facultad, y, en el caso del Hospital Público, de todo su personal.

En tal sentido, el Seminario-Taller, que inicialmente se pensó tan solo llenaría una deficiencia cognitiva de conocimiento, ha demostrado en su aplicación real, su capacidad de enseñar habilidades (análisis de problemas éticos en situaciones clínicas y administrativas), además de promover cambio de actitudes. Esto nos permite afirmar que la ética debe ser enseñada en el nivel vivencial.

Sin embargo, para que tal enseñanza pueda ser asimilada adecuadamente por los estudiantes, resulta indispensable otorgarles una estructura teórica previa que les permita comprender la dinámica de desarrollo y consecuencias de los problemas morales. Creemos que tal estructura fue cubierta en nuestro caso, de manera excelente por la teoría del desarrollo moral del hombre que postula Pedro Ortiz ${ }^{(8,9)}$ y que se expuso en la primera parte del Seminario. Esta teoría plantea que todo el sistema vivo está organizado por alguna clase de información; que ésta determina no solo la estructuración epigenética de las especies, sino también la reestructuración cinética de las mismas; que las clases de información genética, metabólica, neural y psíquica son internas a los seres vivos, y que únicamente los hombres crearon la información social, codificada por fuera de ellos. Esta es la clase de información que cada niño que nace debe incorporarla y codificarla en su neocórtex cerebral, el cual, de esta manera, se convierte en su conciencia. Será pues la actividad consciente la que determine la reestructuración cinética del individuo humano hasta su transformación en una personalidad. Propone, en consecuencia, que las clases de información social que conocemos como principios éticos son, en realidad, aspiraciones éticas que surgieron en el curso de la historia de la sociedad. De estas aspiraciones, las fundamentales son las de solidaridad, de libertad y de justicia, de cuya cabal realización dependerá que la conciencia sea moral; mejor dicho, que sus componentes sentimientos, conocimientos y motivaciones sean de naturaleza moral. La teoría predice la factibilidad del perfeccionamiento moral de la personalidad a través de la educación, así como el desarrollo moral ampliado de toda persona, independientemente de su edad, hasta llegar a ser una persona digna, autónoma e íntegra. Finalmente, la teoría predice que, en tanto esta sociedad no se moralice íntegramente, cada persona debe comprometerse en su moralización, lógicamente después de conocer la múltiple determinación de la inmoralidad vigente.

Por todo ello, la enseñanza de la ética resulta una tarea esencial en la sociedad actual, lo cual se evidencia con mayor dramatismo en los sectores de Salud y Justicia. 
En el primero de ellos, que por profesión nos corresponde, ya constituye preocupación esencial a lo largo de los últimos 40 años, particularmente por el impacto producido por Van Rensselaer Potter con la publicación de su libro Bioethics: Bridge to the future, en $1971^{(10)}$, y el de Clinical Bioethics, de Beauchamp y Childress, en $1979^{(11)}$.

Nuestro trabajo, en el curso de cinco y medio años, apuntó primero a evidenciar cuantitativamente lo que en 2002 nos parecía notorio, el déficit en conocimientos en esta área por parte del cuerpo docente de la Escuela de Medicina y, con ello, la falta de un lenguaje ético común en la institución. Sin embargo, el hecho de que los alumnos de medicina, de manera regular recibieran dos cursos durante la carrera, creaba un desequilibrio en la institución. Por ello pensamos que los profesores debieran compartir un conocimiento común, pero, esta vez, no solo de bioética, sino también de Ética en Salud y Deontología Médica. El proyecto, muy pronto hizo evidente que lo más importante de todo ello se concentraba en los problemas de la moral institucional que como 'atmósfera diaria' debiera ser respirada no solo por los profesores y los alumnos, sino por los miembros del cuerpo administrativo y por todo aquél que ingrese al recinto. Conocemos tan solo de un intento anterior llevado a cabo por Miguel Suazo y su equipo, en el Centro INTEC de Bioética de Santo Domingo, aunque con objetivos y metodologías diferentes ${ }^{(12)}$.

Estos postulados se hicieron evidentes cuando el impacto que fue creando el proyecto al interior de la Facultad de Medicina, a la que pertenece la Escuela de Medicina, se filtró al exterior y fuimos solicitados por los directores de un hospital público a repetir la experiencia con ellos, pero con una finalidad más específica, contribuir a reforzar el ethos institucional y con ello combatir las variables de corrupción, de estrés laboral y de pésimas relaciones entre el personal de la institución que estaban creando un clima de desgobierno inmanejable. La única condición que pusimos para ello fue que asistiera obligatoriamente al curso todo el personal, desde el Director hasta el empleado de menor categoría. Por los resultados inmediatos, evidenciados por los comentarios de los participantes, el beneficio logrado llegó a ser mucho más amplio que el propuesto por el proyecto, pues no solo se benefició la institución con el cambio del clima laboral, sino también las relaciones interpersonales, particularmente por la disminución del estrés laboral. Ello fue posible en la medida que los trabajadores comprendieron la dinámica de los problemas morales, los factores que generaban el alto estrés y que afectaban, en definitiva, su propia salud. Sin embargo, lo realmente impactante fue comprender cómo ante tal situación cada uno de ellos "llevaba una buena cantidad del estrés en su interior" y lo descargaba en su propia familia, al menor estímulo irritante. El cambio de actitudes al que se refiere el personal en sus comentarios, deriva así del proceso de asunción de la propia responsabilidad, de aquella que Etxeberria divide como concepto en: Por uno mismo, Por la obra bien hecha, Como omisión, En el marco institucional y como Respuesta a los otros ${ }^{(13)}$, y de comprender que la ética es, en el fondo, 'un mecanismo de defensa social de supervivencia' que ha usado la humanidad para poder lograr una convivencia saludable, que garantice la continuidad de uno mismo, de la familia y de la especie. La ética surge cuando tenemos al otro u otros al frente de nosotros mismos $\mathrm{y}$, sin poder escapar, debemos, solidariamente, convivir por el bien común que, miles de años atrás, era el bien de la tribu.

Por eso, y enfocando la enseñanza de la ética desde esa perspectiva de importancia fundamental, la pregunta es cómo debe enseñarse, porque parece obvio que esta enseñanza cultural y societaria (formal e informal) viene fracasando.

Por ejemplo, según F. Lolas, es necesario replantear la formación bioética en términos de las 'competencias' más que de ciertos contenidos, como serían:

1. La 'capacidad de formular conflictos morales'.

2. La posibilidad de 'dialogar en forma tolerante' con personas que tienen convicciones y creencias distintas de las propias.

3. La habilidad para 'formular, fundamentar y aplicar principios', entendiendo por tales normas de carácter general que se formulan con base en la experiencia, pero son contrastadas con las consecuencias derivadas de los actos y las decisiones de las personas ${ }^{(14)}$.

Ortiz P (2007), por su lado, propone que la enseñanza de la Ética debe encaminar al estudiante a fin de que asuma el compromiso moral de cumplir con los objetivos siguientes:

1. La transformación moral de las instituciones médicas y de salud, sobre la base de:

a) Un conocimiento de la historia de la moralidad en el mundo y las condiciones de la misma en nuestras instituciones fundamentales.

b) El conocimiento del papel de la Ética filosófica en la historia.

c) La elaboración de estrategias de moralización para optimizar la calidad de la atención médica en el país.

2. El desarrollo moral ampliado de sí mismo, tanto en el pregrado como en el posgrado, fundado en:

a) El conocimiento del desarrollo moral de la personalidad.

b) El conocimiento de la estructura moral de la conciencia, especialmente de las actitudes ante el trabajo, ante los demás y ante sí mismo $^{(15)}$.

En la experiencia lograda en el presente trabajo nos ha parecido importante que en un momento determinado surge la responsabilidad personal y la responsabilidad grupal; es el momento en que el participante deja de culpar a los demás por lo que ocurre en el trabajo y 'toma conciencia' de su papel en el desarrollo de los problemas; es decir, asume su responsabilidad en los hechos y, con ello, abre las puertas para las correcciones adecuadas que podría introducir. Del mismo modo, renace también el sentimiento de solidaridad y la responsabilidad de grupo para hacer algo al respecto. La pregunta es ¿Hasta dónde puede llegar esta reacción ética que parte de las bases de la institución y que debiera modificar el ethos institucional? Por el momento, esta pregunta solo puede ser contestada aproximadamente: "Hasta que las autoridades lo permitan”. El proceso requiere de pleno convencimiento y apoyo político de las autoridades máximas de la institución para hacerlo realmente institucional. En el caso nuestro, como muchas veces ocurre en nuestro país, terminada la primera fase de la intervención y cuando, sobre la base de los resultados, las tratativas generaban nuevas estrategias para su continuación, las autoridades del Hospital fueron cambiadas por decisión política y hubo que interrumpir el proceso.

En conclusión, el trabajo:

1ํ) Evaluó a los profesores de la Escuela de Medicina de la UNMSM, encontrando un deficiente nivel de conocimientos sobre ética, deontología y bioética clínica;

2o) Identificó los problemas éticos más frecuentes en la relación docente-discente y en las sedes docentes, encontrando un rango amplio que iban de problemas leves a preocupantes; 
$3^{\circ}$ ) Construyó, sobre los resultados, un seminario-taller que, una vez validado, fue ofrecido al profesorado como enseñanza básica.

$4^{\circ}$ ) Finalmente se desarrolló y aplicó una estrategia de la moralización y reforzamiento del ethos institucional, que puede ser replicado en otros escenarios.

\section{Palabras Finales}

Por los resultados expuestos, creemos que el valor del presente trabajo debe ser enjuiciado desde varios ángulos:

$1^{\circ}$ Como una nueva técnica para la enseñanza de la ética, más vivencial y basada en los problemas personales que se enfrentan diariamente, antes que como curso únicamente teórico.

2o Como un nuevo método de identificación de problemas morales y laborales que inciden en el ethos institucional y en el estrés laboral.

$3^{-}$Como una nueva estrategia de moralización institucional.

4ํㅡㄹ Como una demostración de cuán importante puede ser una estructura teórica que dé el marco necesario para la asimilación de la enseñanza, como es el caso de la teoría propuesta por Pedro Ortiz, sobre el desarrollo moral del hombre, que hemos utilizado.

Desde esta perspectiva, creemos que la investigación científica tiene una ineludible responsabilidad moral. De acuerdo a Emanuel, ésta debe poseer 'Valor en Sí', vale decir, orientarse a beneficiar a las personas que hayan participado en el estudio y a la sociedad en general ${ }^{(16)}$, ayudándolos a resolver los problemas que generaron el estudio. Nuestro trabajo se enmarca en tal intención.

Finalmente, aunque es obvio que nuestros resultados solo deben ser aceptados como preliminares, nos parece que abren nuevas rutas de indagación en el área de la moralidad/inmoralidad de las instituciones, tarea esencial en nuestro país. Por ello, pensamos que la metodología creada puede posibilitar una ruta de nuevas experiencias en este campo, no solo en instituciones del sector salud sino en las de otros sectores, frecuentemente afectadas en su ethos organizacional por variables de corrupción, de violencia y de estrés laboral. No olvidar que un país presionado por tales factores solo podrá defenderse de ellos y proteger su desarrollo si es que cuenta con instituciones básicas sólidas en las cuales pueda apoyarse.

\section{AGRADECIMIENTOS}

Los autores desean agradecer a los Profesores, Dr. Demetrio Molero Castro y Dr. Salomón Zavala Sarrio, quienes revisaron el texto original y aportaron valiosas sugerencias.

El presente estudio fue apoyado por el Consejo Superior de Investigaciones de la UNMSM. durante los años 2002 y 2003 y financiado desde 2004 a 2007con Códigos de estudio: 020112205, 040112061, 050120211, 060112301, 070120381, con el título "Conocimientos de Ética, Deontologia y Bioética Clínica de los profesores de la Escuela de Medicina de la Universidad Nacional Mayor de San Marcos".

\section{REFERENCIAS BIBLIOGRÁFICAS}

1. Ortiz P. El Sistema de la Personalidad. Lima: Orion; 1994.

2. Perales A. El acto médico: criterios de definición y límites. Diagnóstico. 2001;40(1):46-52.

3. Asociación Latinoamericana de Academias Nacionales de Medicina. Deshumanización de la Medicina y Bioética. Quito: Casa de la Cultura Ecuatoriana "Benjamín Carrión"; 1967.

4. Perales A, Mendoza A, Ortiz P. El mercado profesional como factor de inconducta médica. An Fac Med. 2001;62(2):100-14.

5. von Bertalanffy L. Teoría General de los Sistemas. Buenos Aires: Fondo de Cultura Económica de Argentina; 1991.

6. Orfila-Barreto M. Marco conceptual de la pertinencia educativa en el concepto de la pedagogía crítica. Seminario de Enseñanza de la Bioética. Lima: OPS/OMS; 1999.

7. Dewey J. Experience \& education. The Kappa Delta Pi Lecture Series - Collier Books. New York: Collier Macmillan Publishers; 1938.

8. Ortiz P. Introducción a una psicobiología del Hombre. En: Cuadernos de Psicobiología Social 1. Lima: Universidad Nacional Mayor de San Marcos; 2004.

9. Ortiz P. El nivel consciente de la actividad personal. En: Cuadernos de Psicobiología Social 6. Lima: Universidad Nacional Mayor de San Marcos; 2004.

10. Van Rensselaer P. Bioethics. Bridge to the future. New Jersey: Prentice-Hall INC; 1971.

11. Beauchamp TL, Childress JF. Principles of Biomedical Ethics. New York: Oxford University Press; 1990.

12. Suazo M. Bioética para nuevos. Santo Domingo: Centro INTEC de Bioética/Instituto Tecnológico de Santo Domingo; 2002.

13. Etxeberria $X$. La responsabilidad como eje vertebrador de la ética. Lima: Postgrado de la Facultad de Medicina, UNMSM; 2002.

14. Lolas F. Tercer Simposio Latinoamericano de Bioética y Odontoestomatología. Universidad Peruana Cayetano Heredia. Lima: Postgrado de la Universidad Peruana Cayetano Heredia; 2007.

15. Ortiz P. Ética social para el desarrollo moral de las instituciones educacionales y de salud. Lima: Universidad Nacional Mayor de San Marcos; 2007.

16. Emanuel E. ¿Qué hace que la investigación clínica sea ética? Siete requisitos éticos. En: Pellegrini A, Macklin R (Eds). Investigación en sujetos humanos: experiencia internacional. Washington: OPS/OMSPrograma Regional de Bioética; 1999.

Manuscrito recibido el 12 de febrero de 2008 y aceptado para publicación el 8 de abril de 2008.

\section{Correspondencia:}

Dr. Alberto Perales Cabrera

Av. Javier Prado Oeste, 445. Of. 101

Lima 27, Perú

Correo-e: perales.alberto@speedy.com.pe 